\title{
Molecular Square Dancing in CO-CO Collisions
}

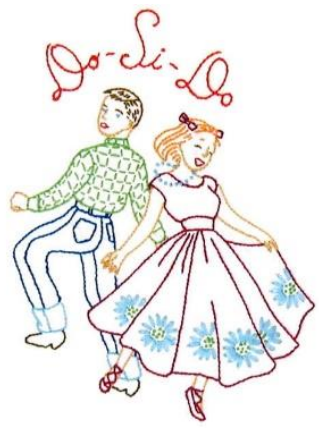

Zhong-Fa Sun ${ }^{1}$, Marc C. van Hemert ${ }^{2}$, Jérôme Loreau ${ }^{3}$, Ad van der Avoird ${ }^{4}$, Arthur G. Suits $^{5}$, David H. Parker*, ${ }^{1}$

1. Department of Molecular and Laser Physics, Institute for Molecules and Materials, Radboud University, Heyendaalseweg 135, 6525 AJ Nijmegen, The Netherlands

2. Leiden University of Chemistry, Gorlaeus Labs. Leiden University, Leiden, The Netherlands

3. KU Leuven, Department of Chemistry, B-3001 Leuven, Belgium,

4. Theoretical Chemistry, Institute for Molecules and Materials, Radboud University, Heyendaalseweg 135, 6525 AJ Nijmegen, The Netherlands

5. Department of Chemistry, University of Missouri, Columbia MO 65211, USA

Corresponding Author. Email: parker@ science.ru.nl

\begin{abstract}
Collisions between two carbon monoxide $(\mathrm{CO})$ molecules resulted in rotational excitation of both species, which were measured simultaneously by advanced experimental methods. An extraordinary energy transfer channel was revealed, combining forward scattering and high rotational excitation of both $\mathrm{CO}$ product molecules, which defied prediction from prevailing models of inelastic scattering. Simulation by quasi-classical trajectories showed that these unusual events start with the $\mathrm{C}$ ends of $\mathrm{CO}$ colliding, with immediate and large transfer from translational to rotational energy. Unlike typical atom-molecule collisions, the two rotating molecules then swing around and depart from the back-to-back $\mathrm{O}$ ends configuration, resulting in forward scattering. Similar effects could occur in other molecule-molecule scattering processes, which are just beginning to be investigated using the velocity map imaging method applied here.
\end{abstract}


If Art follows Nature the square dance call "swing your partner, do-si-do" must have been inspired by dance moves laid out during collision of two $\mathrm{CO}$ molecules, where despite a considerable 'swing' (conversion of translational to rotational energy), both molecules leave the encounter moving forward. Up to now, the dance moves for rotational energy transfer (RET) in atom-molecule scattering have been described as being annoyingly predictable (1): large swings are always accompanied by about-face or backward scattering. Advanced experimental methods, particularly velocity map imaging, VMI, (2), can now expose details of molecule-molecule scattering with resolution sufficient to recognize new collision processes involving unexpected motion, as shown in this report. Although the likelihood of the extraordinary process observed here depends strongly on the initial collision geometry, we show that it is significant, being responsible for production of up to $20 \%$ of high rotational states.

Our current understanding of RET, which is the most probable energy exchange process during a molecular collision, is nearly perfect for atom-diatom collisions, based in detail on accurate fully quantum calculations and in general by simple and precise models that treat the collision as a point mass colliding with a hard shell ellipsoid scaled to the shape of molecule. Along with predicting the directionality of scattering, these models also lead to the exponential energy gap law (EGL) (3) where the probability of collision induced transfer is found to be proportional to $\exp (-C \Delta E)$, with $\Delta E$ the energy difference between the ground and excited rotational states, and $C$ is a constant. The EGL ascribes a much lower probability of rotational excitation to higher $j$ levels, where $j$ is the rotational quantum number. A key question is whether this picture always applies to moleculemolecule scattering.

Because a single collision can cause rotational excitation of both collision partners in moleculemolecule interactions, the ideal RET experiment provides for each possible product-pair the directionality of scattering, also known as the pair-correlated differential scattering cross section (PC-DCS). This measurement is now possible when using crossed molecular beam scattering (4) combined with VMI, where each molecular beam has a narrow (5) or ultra-narrow and controlled (6) velocity distribution and contains only the lowest $(j=0)$ rotational state of the molecule of interest. Nascent product molecules in a chosen final ( $\left.j^{\prime}\right)$ state are then converted to ions by resonant laser ionization and their velocity distribution is mapped using VMI with high speed and angular resolution onto a two-dimensional image (7-9). Our study explores (nearly) identical 
molecules scattering, which offers conceptual and computational advantages over mixed molecules. We characterize RET in the collision system ${ }^{13} \mathrm{CO}(j=0)+{ }^{12} \mathrm{CO}(j=0) \rightarrow{ }^{13} \mathrm{CO}(j)+$ ${ }^{12} \mathrm{CO}\left(j{ }^{\prime}\right)$, where for practical reasons specific $j$ ' rotational quantum levels of ${ }^{13} \mathrm{CO}$ product molecules are detected. Besides being of fundamental interest, $\mathrm{CO}-\mathrm{CO}$ scattering is relevant in the pure $\mathrm{CO}$ atmosphere of exotic exoplanets (10) and in cometary atmospheres, where $\mathrm{CO}$ is the second most abundant molecule after $\mathrm{H}_{2} \mathrm{O}$. $\mathrm{CO}$ has a small dipole moment, and forms a dimer with two stable, end-on anti-parallel geometries with relatively small binding energies of about $125 \mathrm{~cm}^{-1}(11)$. The CO-CO center-of-mass distance is substantially larger for the geometry with the $\mathrm{C}$-ends closer than when the $\mathrm{O}$-ends are closer, because the $\mathrm{C}$-end is effectively larger than the O-end. We show that the latter property also has interesting effects in $\mathrm{CO}-\mathrm{CO}$ collisions.

We report here scattering of ${ }^{12} \mathrm{CO}(j=0)$ with ${ }^{13} \mathrm{CO}(j=0)$ to form ${ }^{13} \mathrm{CO}(j ;=15)$, where the experiment yields the PC-DCS for each $j$ " level of ${ }^{12} \mathrm{CO}(j "=0,1,2 \ldots$, up to $j "$ max $)$ paired with ${ }^{13} \mathrm{CO}(j$ ' $=15)$. However, in this so-called 'heavy-heavy' collision system where the CO rotation energy spacing is much smaller than the collision energy, only partial (but sufficient) resolution of the product-pair DCSs was possible. Note that full resolution of pair-correlated DCSs for collisions of $\mathrm{NO}$ with $\mathrm{O}_{2}(12)$ and $\mathrm{ND}_{3}-\mathrm{D}_{2}(13)$ has been recently reported, both at lower collision energy than the present system.

Crossed-beam scattering with identical molecules poses severe overlap problems that were solved here using a beam of pure ${ }^{13} \mathrm{CO}$ and selective ${ }^{13} \mathrm{CO}$ ionization. Moreover, we detected final states that were too high in energy to be populated by collision of ${ }^{13} \mathrm{CO}$ with the carrier gas used for rotational state cooling of CO in the partner beam, as described in the Supplementary Materials $(\mathrm{SM})$ section. The conditions that avoided overlap allowed detection of ${ }^{13} \mathrm{CO}(j '>10)$ final states from CO-CO scattering at $1460 \mathrm{~cm}^{-1}$ collision energy, which for a 'heavy-heavy' system has too many open channels at present for analysis by fully quantum theory. Quasi-classical trajectory (QCT) analysis on an accurate CO-CO potential energy surface $(14,15)$, proven to be reliable for $\mathrm{CO}-\mathrm{He}$ and $\mathrm{CO}-\mathrm{Ar}$ collisions in previous publications $(16,17)$, was used here to simulate the scattering process. The QCT results were confirmed at lower collision energy by fully quantum close-coupling $(\mathrm{CC})$ calculations and compared with those from a previous fully quantum theory study on CO-CO (18) in the SM. 
Details of the experiment and analysis methods are presented in the SM. Raw velocity map images of ${ }^{13} \mathrm{CO}\left(j^{\prime}=15\right)$ molecules formed by scattering with $\mathrm{CO}$ and with $\mathrm{N}_{2}$ for comparison (Fig. S1) showed an obvious difference of 'extra' signal in the forward scattering region around high $j$ " $\approx j$, labelled from hereon as forward-scattered symmetric excitation (FSSE), which was present for ${ }^{13} \mathrm{CO}-\mathrm{CO}$ but missing for ${ }^{13} \mathrm{CO}-\mathrm{N}_{2}$. After correcting the raw images for ionization laser polarization sensitivity and the density-flux transform, the experimental data is plotted in Fig. 1 in $(j ", \theta)$ coordinates, where the image radius is converted to $j$ " position. FSSE signal peaked at $j$ " $=15$, was seen only in the $\mathrm{CO}+\mathrm{CO}$ data, as indicated by an arrow in both the experimental plot (left panel of Fig. 1) and the corresponding theoretical prediction (right panel). An explanation for why FSSE is absent for the kinematically similar $\mathrm{CO}+\mathrm{N}_{2}$ system was provided by the QCT analysis, as described later in the text. 


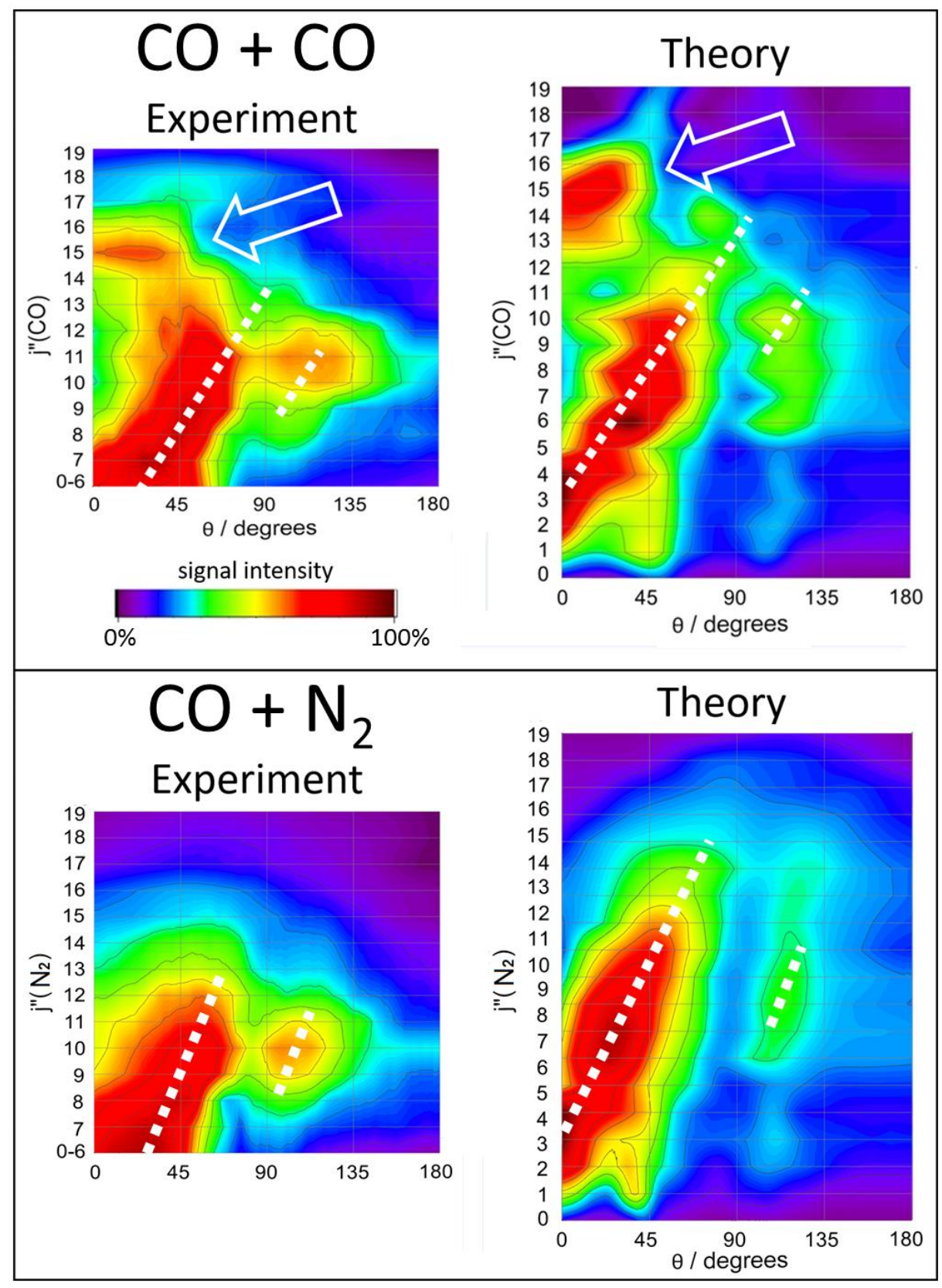

Fig. 1. Contour plot of experimental and theoretical pair-correlated DCSs for ${ }^{13} \mathrm{CO}+\mathrm{CO}$ and ${ }^{13} \mathrm{CO}+\mathrm{N}_{2}$ inelastic scattering to the $j,\left({ }^{13} \mathbf{C O}\right)=\mathbf{1 5}$ final state. Left panels: Partially-resolved experimental pair-correlated DCSs with scattering angle on the horizontal axis and vertical axis indicates the final rotational state of the $\mathrm{CO}$ or $\mathrm{N}_{2}$ co-product, $j$ ' $\left(\mathrm{CO}\right.$ or $\left.\mathrm{N}_{2}\right)$, which ranges from the low states $\left(j\right.$ ' $\left(\mathrm{CO}\right.$ or $\left.\left.\mathrm{N}_{2}\right)=0-6\right)$, at the bottom, to the higher states at the top up to $j{ }^{\prime \prime}{ }_{\max }\left(\mathrm{CO}\right.$ or $\left.\mathrm{N}_{2}\right)=19$. The $j "\left(\mathrm{CO}\right.$ or $\left.\mathrm{N}_{2}\right)=0-6$ signals are not resolved. Right panels: Theoretical pair-correlated DCSs fully resolved in individual final rotational state of co-product $\mathrm{CO}$ or $\mathrm{N}_{2}, j$ " ( $\mathrm{CO}$ or $\mathrm{N}_{2}$ ) convoluted over the experimental uncertainty. An arrow in both panels for ${ }^{13} \mathrm{CO}+\mathrm{CO}$ indicates the region of the unexpected forward scattered high $j^{\prime \prime} \approx j$ ' signal, which is not observed for the kinematically similar ${ }^{13} \mathrm{CO}+\mathrm{N}_{2}$ scattering. 
Dashed diagonal lines shown in Fig. 1 indicate regions of atom-molecule type behavior for inelastic scattering, where conversion of translational energy to an increasingly larger amount of rotational energy required an increasingly 'head-on' collision with the target molecule, and thus a smaller impact parameter (the distance of closest approach that would have occurred without any interaction). Small impact parameter collisions result in scattering towards increasingly larger (backward) angles. The long diagonal dashed line follows the ridge of the primary rainbow (a build-up of trajectories that pass threshold for rotational excitation (1)) while the shorter dash, at larger scattering angles, follows the expected secondary rainbow.

Fig. 1 shows satisfactory agreement, including the FSSE signal, between experiment and QCT. A full description of the QCT results including the FSSE $v s$. EGL relative yields in CO-CO RET at 1460 and $500 \mathrm{~cm}^{-1}$ collision energy is presented in the SM section. The $j^{\prime}, j^{\prime \prime}=15,15$ pair correlated differential cross section with forward scattering angles of $30^{\circ}$ or less, originated predominantly from trajectories with an impact parameter between 3.0 and 3.5 $\AA$. More than half of the trajectories leading to forward scattering were very similar to the prototypical trajectories shown in movie M1 in the SM, and sketched in Fig. 2. For comparison, movie M2, shows nonFSSE collisions producing $(5,15)$ products. 


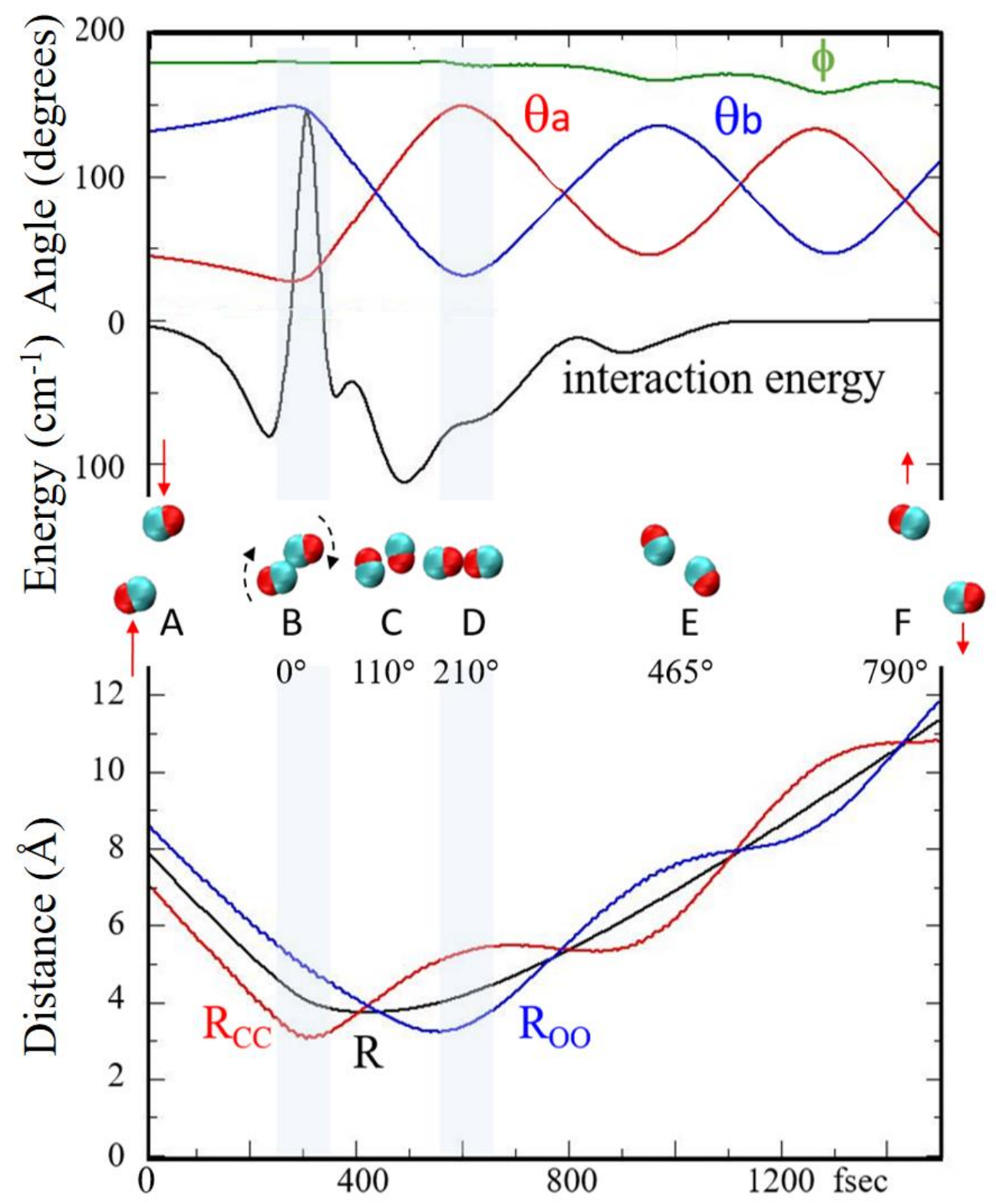

Fig. 2. Trajectory details for two colliding $\operatorname{CO}(j=0)$ molecules, ending with both in $j=15$. $\mathrm{R}_{\mathrm{CC}}$, $\mathrm{R}_{\mathrm{Oo}}$ are internuclear distances, and $\mathrm{R}$ is the center-of-mass distance between the two diatoms. The dihedral angle $\phi$ is $180^{\circ}$ when the molecular axes are coplanar and anti-parallel, and $\theta_{\mathrm{a}}, \theta_{\mathrm{b}}$ are the angles between the $\boldsymbol{R}$ vector and the bond directions of molecule a (traveling up in the figure) and b (down), respectively. In the upper panel energy $\left(\mathrm{cm}^{-1}\right)$ and angle (degrees) have the same vertical axis spacing. The collision geometry is shown in the middle of the figure for positions A-F, corresponding to times of 0 , 275, 450, 600, 1000, and $1500 \mathrm{fs,} \mathrm{respectively.} \mathrm{Molecular} \mathrm{rotation} \mathrm{begins} \mathrm{at} \mathrm{position} \mathrm{B)} \mathrm{and} \mathrm{reaches} \mathrm{angle}$ values shown under each position. Collision energy is $1460 \mathrm{~cm}^{-1}$. See text and SM (M1) for more details. 
Three unusual features in Fig. 2 characterized FSSE: a) initial collision geometry, b) abruptness of RET, and c) the do-si-do movement. The collision geometry is shown in the middle region of Fig. 2 as a series of stills at time positions A-F in the figure, from a VMD (19) visualization of a prototypical $\left(j, j^{\prime}{ }^{\prime}=15,15\right)$ trajectory. The most effective initial geometry was where the two Cends collide, with an impact parameter of $3.2 \AA$. Forward scattering was most probable when both the molecular axes were in the same collision plane $(\phi \approx 0)$ defined with respect to the internuclear separation vector $\boldsymbol{R}$. The interaction energy curve in Fig. 2 showed that collision at point B, $240 \mathrm{fs}$, was abrupt and accounted for the translation to rotational energy transfer, with both molecules rotating individually and in the same sense. Most striking in Fig. 2 was the relatively constant value of $\mathrm{R}$ from $\sim 240$, to $\sim 600 \mathrm{fs}$, which was roughly half of a $\mathrm{CO}(j=15)$ rotational period. The average speed here was $\geq 492 \mathrm{~m} / \mathrm{s}$, so near-zero recoil means the molecules swing around each other with little repulsion/additional torque to reach the O-end facing or back-to-back ( $d o-s i-d o$ ) configuration. In square dancing, a charm of the $d o$-si-do is that both pairs of feet point outwards, avoiding tripping. In the case of CO-N 2 , FSSE was not possible due to tripping by the ellipsoidal $\mathrm{N}_{2}$ partner. Two ovoids, colliding first at their large ends, are needed for FSSE. As shown in Fig. S5c, FSSE is not due to the displaced center of mass in $\mathrm{CO}$, but was rather a size (electronic volume) effect of the potential energy surface. Following the $d o$-si-do, which allows RET far beyond EGL predictions, the rotationally excited molecules departed in the forward scattering direction.

FSSE collisions showed two sequential interactions, where the first, $\mathrm{C}-\mathrm{C}$ end, collision resulted in translation to rotation energy transfer while the second interaction at the $\mathrm{O}-\mathrm{O}$ end reoriented the recoil direction of the two molecules as they separated, without changing the rotational energy. Viewing the process in reverse, the O-O end interaction first swings the molecules around closer to each other so that when they slammed together on the C-C ends, all rotation energy was up-converted to faster linear motion. QCT yielded a final impact parameter (i.e., for the reverse reaction) of $b_{f}=4.0 \pm 0.1 \AA$, i.e., $20 \%$ larger than the initial $b_{i}=3.2 \AA$, which was a simple result of conservation of energy and angular momentum when starting with two $j=0$ molecules. As $\boldsymbol{R}$ expanded by $0.8 \AA$ during the collision, it also rotated, causing the final recoil direction to turn from sideways towards forward as illustrated in the cartoon of Fig. 3. 


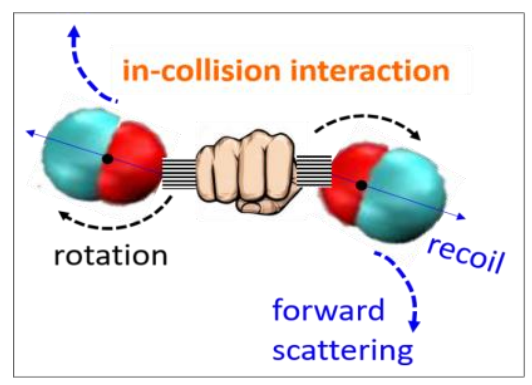

Fig. 3. Cartoon of scattering angle reorientation. With the collision geometry at position A in Fig. 2, or its mirror image, both $\mathrm{CO}$ molecules rotate in the same sense and when the intermolecular interaction during the collision couples with this rotational motion, the recoil direction will rotate towards the forward direction.

At lower collision energy $\left(500 \mathrm{~cm}^{-1}\right)$, QCT predicted the same FSSE process, peaking at $\left(j{ }^{\prime}, j{ }^{\prime \prime}\right)=$ $(8,8)$, with forward scattering being most probable at $b_{i}=3.0 \AA$. FSSE occurred for both collision energies with roughly $60 \%$ conversion of translation to rotational energy. Fully quantum CC calculations at $500 \mathrm{~cm}^{-1}$ confirmed a significant amount of rotational excitation for near symmetric excitation, peaking even closer to forward scattering than the QCT results, Fig. S8. $\mathrm{CC}$ calculations also revealed a propensity for collisions resulting in even values of final $j$, Fig. $\mathrm{S} 7$, reflecting the nearly homonuclear diatomic nature of $\mathrm{CO}$.

Pair-correlated excitation cross sections observed previously for $\mathrm{NO}-\mathrm{O}_{2}$ collisions (12) also deviate from EGL behavior in that strong excitation in both the $\mathrm{NO}$ and $\mathrm{O}_{2}$ molecules is preferred over excitation in one collision partner only, even though the total amount of energy transfer is higher. Opacity function analysis showed that the inelastic channels for high rotational state excitation were governed by short-ranged head-on collisions and that only the long-ranged contributions follow 'normal' behavior (12).

$\mathrm{CO}-\mathrm{CO}$ is not a particularly unusual collision system. We suggest FSSE-like behavior is also possible in mixed molecule scattering systems that match the criteria found here: a) large ends colliding first, b) an abrupt energy transfer coupled with a swing around towards the small end configuration before departure. Potential energy surfaces with minor topological features compared to the collision energy could allow the pathway observed here for CO-CO scattering. As molecule-molecule scattering is explored in higher detail with new methods, more unusual molecular dance moves could be expected. 


\section{References}

1. R. J. Marsh, A. J. McCaffery, Chem. Phys. Lett. 335, 134-139 (2001).

2. A. T. Eppink, D. H. Parker, Rev. Sci. Instrum. 68, 3477-3484 (1997).

3. J. C. Polanyi, K. B. Woodall, J. Chem. Phys. 56, 1563-1572 (1972).

4. Y. T. Lee, J. D. McDonald, P. R. LeBreton, D. R. Herschbach, Rev. Sci. Instrum. 40, 1402-1408 (1969).

5. B. Yan et al., Rev. Sci. Instrum. 84, 023102 (2013).

6. S. Y. T. van de Meerakker, H. L. Bethlem, N. Vanhaecke, G. Meijer, Chem. Rev. 112, 4828-4878 (2012).

7. G. Sarma, S. Marinakis, J. J. ter Meulen, D. H. Parker, K. G. McKendrick, Nat. Chem. 4, 985-989 (2012).

8. S. N. Vogels et al., Phys. Rev. Lett. 113, 263202 (2014).

9. A. von Zastrow et al., Nat. Chem. 6, 216-221 (2014).

10. K. B. Sheppard et al., Astrophys. J. 850, L32 (2017).

11. G. W. M. Vissers, A. Heßelmann, G. Jansen, P. E. S. Wormer, A. van der Avoird, J.

Chem. Phys. 122, 054306 (2005).

12. Z. Gao et al., Nat. Chem. 10, 469-473 (2018).

13. Z. Gao, J. Loreau, A. van der Avoird, S. Y. T. van de Meerakker, Phys. Chem. Chem.

Phys. 21, 14033 (2019).

14. G. W. M. Vissers, P. E. S. Wormer, A. van der Avoird, Phys. Chem. Chem. Phys. 5, 4767-4771 (2003).

15. L. A. Surin et al., J. Phys. Chem. A 111, 12238-12247 (2007).

16. L. Song et al., J. Phys. Chem. A 119, 12526-12537 (2015).

17. Z. F. Sun et al., Phys. Chem. Chem. Phys. 21, 9200-9211 (2019).

18. S. A. Ndengué, R. Dawes, F. Gatti, J. Phys. Chem. A 119, 7712-7723 (2015).

19. W. Humphrey, A. Dalke, K. Schulten, J. Mol. Graph. 14, $33-38$ (1996).

20. Z. F. Sun, A. von Zastrow, D. H. Parker, J. Chem. Phys. 147, 013909 (2017).

21. A. G. Suits et al., J. Phys. Chem. A 119, 5925-5931 (2015).

22. D. K. Hoffman, J. W. Evans, D. J. Kouri, J. Chem. Phys. 80, 144-148 (1984).

23. M. Brouard et al., J. Chem. Phys. 138, 104309 (2013).

24. M. Brouard et al., J. Chem. Phys. 146, 204304 (2017).

25. H.-J. Werner, P. J. Knowles, G. Knizia, F. R. Manby, M. Schütz, WIREs Comput. Mol. Sci.2, 242-253 (2012).

26. M. C. van Hemert, J. Chem. Phys. 78, 2345-2354 (1983).

27. X. Hu, W. L. Hase, T. Pirraglia, J. Comput. Chem. 12, 1014-1024, (1991).

28. J. A Coxon, P. G. Hajigeorgiou, J. Chem. Phys. 121, 2992-3008 (2004).

29. M. P. d. Miranda, F. J. Aoiz, L. Bañares, V. S. Rábanos, J. Chem. Phys. 111, 5368-5383 (1999).

30. R. Dawes, X. G. Wang, T. Carrington, J. Phys. Chem. A 117, 7612-7630 (2013).

31. Sun, Zhongfa et al. (2020), Molecular Square Dancing in CO-CO Collisions, Dryad, Dataset, https://doi.org/10.5061/dryad.80gb5mkp0 



\section{ACKNOWLEDGMENTS}

We thank B. v.d Meerakker, G. C. Groenenboom. and R. J. A. Scheidsbach for discussion. R. J. A. Scheidsbach, C. K. Bishwakarma, G. Sarma and L. Gerritsen developed the experimental methods used in this work.

Funding:

The experimental part of this work was funded by the Dutch National Science Foundation (NWO) under grant number TOP 715.013.002, and the Dutch Astrochemistry Network DANII under grant number 648.000.024. AGS acknowledges support from the US National Science Foundation (NSF) under award number CHE-1955239.

\section{Author contributions:}

The experimental work and data analysis was carried out by ZFS, AGS, and DHP. Theoretical calculations were done by $\mathrm{MvH}, \mathrm{JL}$, and AvdA. All authors contributed to interpreting the results and writing the manuscript.

Competing Interests: None declared.

Data and Materials availability: All data are available in the main text or in the supplementary materials and at Dryad (31).

\section{SUPPLEMENTARY MATERIALS}

Experimental Methods

Theoretical Methods

Detailed comparison of experiment and theory

Fig. S1 to S8

Table S1

Data S1

Movies M1 and M2 


\section{Supplementary Materials for}

\section{Molecular Square Dancing in CO-CO Collisions}

Zhong-Fa Sun ${ }^{1}$, Marc C. van Hemert ${ }^{2}$, Jérôme Loreau ${ }^{3}$, Ad van der Avoird ${ }^{4}$, Arthur G. Suits ${ }^{5}$, David H. Parker*, ${ }^{1}$

1. Department of Molecular and Laser Physics, Institute for Molecules and Materials, Radboud University, Heyendaalseweg 135, 6525 AJ Nijmegen, The Netherlands

2. Leiden University of Chemistry, Gorlaeus Labs. Leiden University, Leiden, The Netherlands

3. Department of Chemistry, KU Leuven, Celestijnenlaan 200F, Heverlee, Belgium

4. Theoretical Chemistry, Institute for Molecules and Materials, Radboud University, Heyendaalseweg 135, 6525 AJ Nijmegen, The Netherlands

5. Department of Chemistry, University of Missouri, Columbia MO 65211, USA

*Corresponding Author: Email: parker@ science.ru.nl

\section{This PDF file includes:}

Experimental Methods

Theoretical Methods

Detailed comparison of experiment and theory

Fig. S1 to S9

Table S1

Data and materials availability

Caption for Data S1

Caption for movies M1 and M2

Other Supplementary Materials for this manuscript include:

Data S1

Movies M1 and M2 


\section{Experimental Methods}

A detailed description of the crossed-beam imaging apparatus with variable molecular beam crossing angle can be found in Refs (16) and (17); a short overview concerning the CO-CO scattering experiment producing $j^{\prime}=15$ products is given here. Publications describing the full set of $\mathrm{CO}-\mathrm{CO}$ and $\mathrm{CO}-\mathrm{N}_{2}$ data and analysis are in preparation.

Signal overlap arises because scattering took place between two $\mathrm{CO}$ molecules, each in the $(j=$ 0 ) initial state, prepared by seeding $\mathrm{CO}$ in a supersonic rare gas expansion. Rotationally excited $\mathrm{CO}$ could thus arise from three scattering processes: a) $\mathrm{CO}$ (beam 1) $+\mathrm{CO}$ (beam 2); b) $\mathrm{CO}$ (beam 1) + rare gas(beam 2), and c) $\mathrm{CO}$ (beam 2) + rare gas(beam 1); producing three different images that lined up along their common relative velocity direction. To avoid overlap from signals b) and c), the use of carrier gases with a large speed and mass difference was the first necessary step. A series of trial experiments favored the primary (i.e., detected molecule) beam 1 with $5 \%{ }^{13} \mathrm{CO}$ seeded in Ar gas (stagnation pressure 1.2 bar) and secondary beam 2 with normal (n-)CO (99\% $\left.{ }^{12} \mathrm{CO}, 1 \%{ }^{13} \mathrm{CO}\right) / \mathrm{He}$ at a stagnation pressure of 1.2 bar. Both beams were formed with a Nijmegen pulsed valve (NPV)(5). Pure ${ }^{13} \mathrm{CO}$ would ideally eliminate signal c). However, $\mathrm{n}-\mathrm{CO}$ in beam 2 contains $\sim 1 \%{ }^{13} \mathrm{CO}$, and the cross section for collision with $\mathrm{Ar}$ in beam 1 is rather large, creating a large-diameter, weak CO-Ar image that underlies signal a). Signal b) remained a major problem, however, and could only be eliminated by observing final $\mathrm{CO}(j$ ') products from a) that were not accessible at the collision energy available for b). Changing the beam crossing angle to $70^{\circ}$ lowered the $\mathrm{CO}+\mathrm{He}$ collision energy to around $360 \mathrm{~cm}^{-1}$; this limited rotational excitation of $\mathrm{CO}$ to $j^{\prime}<13$. Therefore, by probing higher-j' $\mathrm{CO}$ products, the $\mathrm{CO}+\mathrm{He}$ image disappeared completely, leaving only the desired bi-molecular scattering signal a). Both pulsed valves worked at $10 \mathrm{~Hz}$ with pulse duration of about $50 \mu \mathrm{s}$ at the crossing volume of $3 \times 3 \times 3 \mathrm{~mm}^{3}$. The main experimental data was taken at a beam crossing angle of $70^{\circ}$ where the mean velocities of the primary and secondary beams were $\mathrm{v}\left({ }^{13} \mathrm{CO} / \mathrm{Ar}\right) \approx 670 \mathrm{~m} \cdot \mathrm{s}^{-1}, \mathrm{v}\left({ }^{12} \mathrm{CO} / \mathrm{He}\right) \approx 1660 \mathrm{~m} \cdot \mathrm{s}^{-1}$, resulting in mean collision energies of ${ }^{13} \mathrm{CO}+{ }^{12} \mathrm{CO},{ }^{12} \mathrm{CO}+\mathrm{Ar}$ and ${ }^{13} \mathrm{CO}+\mathrm{He}$ systems to be $1460 \mathrm{~cm}^{-1}, 1680$ $\mathrm{cm}^{-1}$ and $360 \mathrm{~cm}^{-1}$ respectively.

REMPI spectra of the primary ${ }^{13} \mathrm{CO}$ beam and secondary ${ }^{12} \mathrm{CO}$ beam were taken to estimate the rotational temperatures: the ${ }^{13} \mathrm{CO} / \mathrm{Ar}$ beam was $\sim 1.5 \mathrm{~K}$, giving a population of $j=0$ about $90 \%$ and of $j=1$ about $10 \%$. Meanwhile, more than $95 \%$ of the molecules in the $\mathrm{n}-\mathrm{CO} / \mathrm{He}$ beam 
populated in the lowest $j=0,1$ states, corresponding to a rotational temperature of $3 \mathrm{~K}$. Under collision conditions, depletion of the parent ${ }^{13} \mathrm{CO}$ beam was estimated to be less than $5 \%$ and was not detectable within our $\mathrm{S} / \mathrm{N}$ limits, ensuring single inelastic collision conditions.

The scattered CO product after collision was state-selectively ionized by either VUV REMPI at $\lambda \approx 154 \mathrm{~nm}$ as described in detail in $\operatorname{Ref}(20)$, or by $(2+1)$ REMPI at $\lambda \approx 215 \mathrm{~nm}$ employing the $E^{1} \Pi-X^{1} \Sigma^{+}(0,0)$ transition of CO. 2+1 REMPI is relatively insensitive to product polarization due to saturation effects and was used in trial experiments with a counter-propagating (head-on) geometry in order to optimize the experimental conditions. VUV REMPI is fully polarization sensitive, but spectral congestion and/or accidental overlap with ${ }^{12} \mathrm{CO}$ REMPI lines limits the probed ${ }^{13} \mathrm{CO}\left(j^{\prime}\right)$ states to $j{ }^{\prime}=15,17,19,22$, and 23 .

$\mathrm{CO}^{+}$ions were projected by the VMI ion optics onto a two-dimensional chevron MCP /phosphor screen detector and mass-selected by turning the multichannel plate (MCP) detector on at the proper arrival time. Typically, images built up from 100,000 laser shots were recorded by a charge coupled device (CCD) camera and transferred to a computer where they were processed and averaged with DaVis software (LaVision) in the event counting mode. Images were accumulated with the polarization direction of the VUV laser parallel and perpendicular to the scattering plane. Following the procedure given in Ref (21), partial integration of the outer radii or a stripe through the two images yielded sufficient information to directly determine parameters describing alignment of the product molecular rotation induced by the collision. Because these parameters were very similar to those measured for $\mathrm{CO}-\mathrm{Ar}$ (17) and agreed well with predictions from a simple classical model (22) we presented alignment corrected images in Fig. S1. These agreed well with the (lower quality) 2+1 REMPI images, which were not alignment sensitive.

Raw velocity map images of ${ }^{13} \mathrm{CO}\left(j^{\prime}=15\right)$ molecules scattered by $\mathrm{CO}$ (panel a) and $\mathrm{N}_{2}$ (panel b) for comparison, the key results for this study, are shown in Fig. S1. Two raw images taken with the laser polarization parallel and perpendicular to the scattering plane were summed for illustration in Fig. S1 in order to diminish polarization effects (23). CO scattering products were formed as nestled three-dimensional velocity spheres whose radius depends on the amount of translation to rotation energy transfer; $100 \%$ would result in a dot at the center of the image disc, which results from projecting the velocity spheres by an electrostatic lens set to VMI conditions onto a two dimensional charged particle imaging detector. 


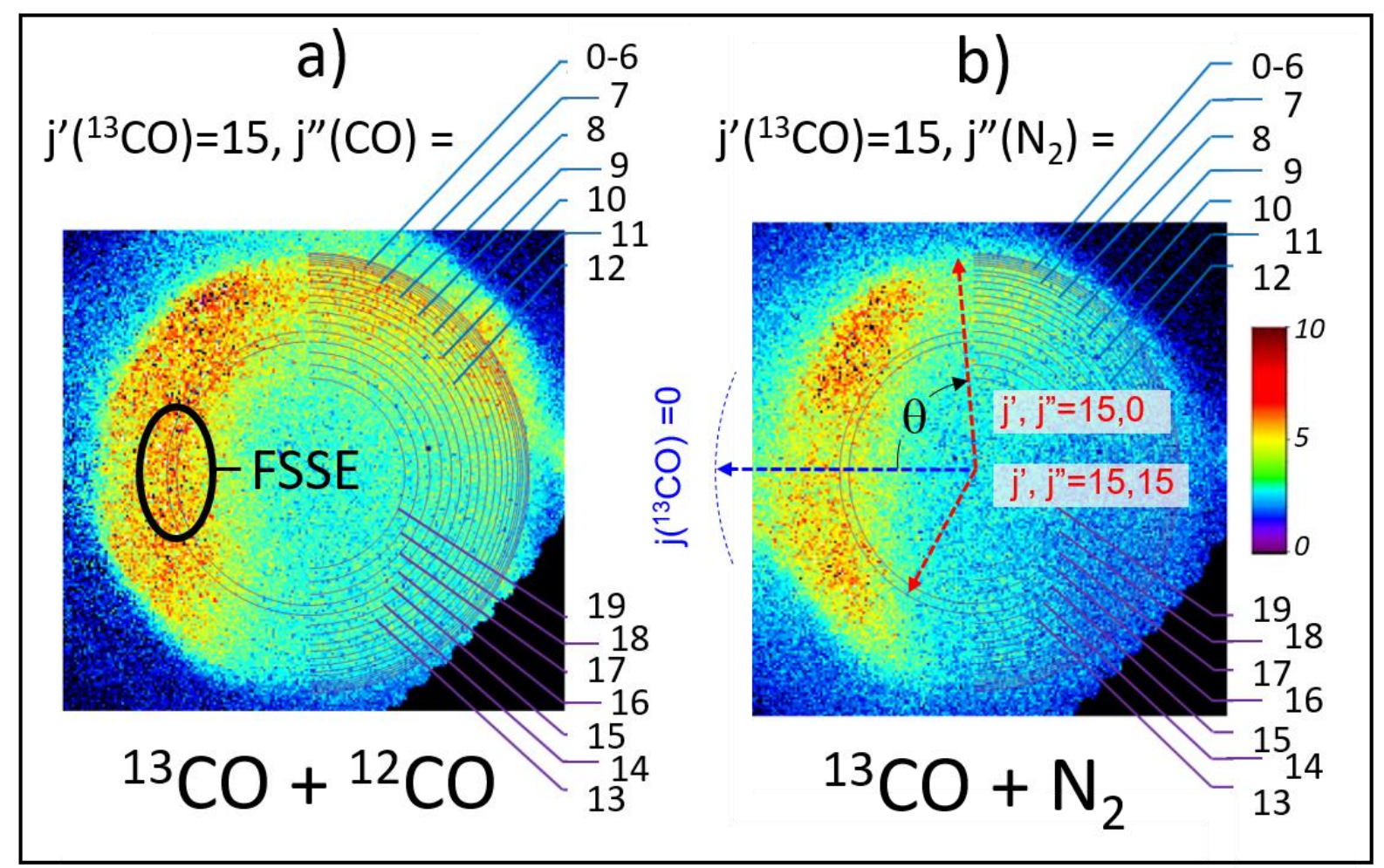

Figure S1: Experimental raw velocity map images of ${ }^{13} \mathrm{CO}\left(j^{\prime}=15\right)$ molecules scattered by $\mathrm{CO}$ (panel a) and $\mathrm{N}_{2}$ (panel b) for comparison. The position of 'extra' signal in $\mathrm{CO}-\mathrm{CO}$ scattering is labeled FSSE in panel a).

A color bar is shown in Fig. S1 for conversion to signal level. The initial velocity of the ${ }^{13} \mathrm{CO}\left(j^{\prime}=0\right)$ beam is shown by a blue dashed arrow in Figure $\mathrm{S} 1 \mathrm{~b}$, the same arrow applies for Fig. S1a. Forward scattered products $\left(\theta=0^{\circ}\right)$ follow this arrow while backward scattered products $(\theta$ $\left.=180^{\circ}\right)$ appeared in the opposite direction on the image. Products of purely elastic scattering $(j=$ $\left.j{ }^{\prime}=j^{\prime \prime}=0\right)$ were confined to the dashed blue circle in Figure S1b. For the data shown here, ${ }^{13} \mathrm{CO}\left(j{ }^{\prime}\right.$ = 15) was detected and concentric circles imposed on the right half of each image in Fig. S1b corresponded to the radial positions of the $\left({ }^{13} \mathrm{CO} j^{\prime}=15, \mathrm{~N}_{2} j^{\prime \prime}\right)$ product pairs. The largest halfcircle with radius marked by a nearly vertical dashed red line in Figure $\mathrm{S} 1 \mathrm{~b}$ corresponded to $\left(j{ }^{\prime}=\right.$ $15, j "=0)$, but the small energy spacing for low $j$ " quantum numbers resulted in overlap of the $j$ " $=0-6$ rings. A second dashed red arrow pointing downwards indicates the radial position of the $j$ " $=15$ final products. To guide the eye, the $j "=15$ and 16 rings were drawn in Figure S1 as full circles, and the region of forward scattering symmetric excitation, FSSE, was indicated in panel a). The energetic limit for $1460 \mathrm{~cm}^{-1}$ collision energy for ${ }^{13} \mathrm{CO}-\mathrm{CO}$ corresponded to $\left(j '=15, j^{\prime \prime}=\right.$ 23). Half-circles for CO coproducts were superimposed on Figure S1a. Due to the similarity in 
rotational constants $\left(\mathrm{B}_{\mathrm{CO}}=1.94 \mathrm{~cm}^{-1}, \mathrm{~B}_{\mathrm{N} 2}=1.99 \mathrm{~cm}^{-1}\right)$ the product-pair circles for both molecules differ visually only at the highest $j$ " values.

A set of procedures described in Refs. $(17,21)$ were followed to fully simulate the CO-CO polarization dependent scattering images. Correction of the imaging signal from density to flux, and extraction of collision induced alignment information was carried out, but because the extraordinary channel described in the text did not show special alignment effects within the signal to noise ratio of the data, the alignment-free moment was extracted for analysis of the radiusdependent angular distribution of the images, i.e., the $\left(j^{\prime}=15, j\right.$ ') pair-correlated scattering data using an onion-peeling inversion program similar to that in Ref. (24). The results of this analysis are shown as Fig. 1 in the main text.

\section{Theoretical Methods}

A new intermolecular potential energy surface (PES) of CO-CO system that is optimized to $>1500$ $\mathrm{cm}^{-1}$ was calculated in coupled cluster singles and doubles with perturbative triples $[\mathrm{CCSD}(\mathrm{T})]$ calculations with an augmented quadruple zeta (AVQZ) basis using the Molpro program (2012 version)(25). All points on the PES, i.e., the interaction energies V(Q) were derived from according to the following formula:

$$
\mathrm{V}(\mathrm{Q})=\mathrm{V}_{\mathrm{AB}}(\mathrm{Q})-\mathrm{V}_{\mathrm{A}}(\mathrm{Q})-\mathrm{V}_{\mathrm{B}}(\mathrm{Q})
$$

Here $\mathrm{Q}$ stands for all intra- and inter-molecular coordinates in the collision complex. $\mathrm{V}_{\mathrm{AB}}$ is the $\operatorname{CCSD}(\mathrm{T})$ energy of the collision complex and $\mathrm{V}_{\mathrm{A}}$ and $\mathrm{V}_{\mathrm{B}}$ are the $\operatorname{CSS}(\mathrm{T})$ energies of the collision partners (i.e., ${ }^{13} \mathrm{CO}$ and $\mathrm{CO}$ monomers). The full basis set for the collision complex was also used for the monomers in order to compensate for the basis set superposition error (BSSE).

The mass of ${ }^{13} \mathrm{CO}$ and normal $\mathrm{CO}$ was taken to be $28.998270 \mathrm{u}$ and $27.9949150 \mathrm{u}$, respectively and the bond lengths were kept fixed at their equilibrium distance $r_{e}=2.132 a_{0}$. With fixed monomer bond length, the PES of the collision complex depends on four coordinates: the length of $\mathbf{R}(R)$, and the angles $\Theta_{\mathrm{A}}, \Theta_{\mathrm{B}}$ and $\Phi$, where $\mathbf{R}$ is the vector pointing from the center of mass of monomer $\mathrm{A}$ to that of $\mathrm{B}, \Theta_{\mathrm{A}}$ and $\Theta_{\mathrm{B}}$ are angles between the vector $\mathbf{R}$ and the vectors $\mathbf{r}_{\mathrm{A}}$ and $\mathbf{r}_{\mathrm{B}}$ pointing from the $\mathrm{O}$-atom to the $\mathrm{C}$-atom in both monomers, and $\Phi$ is the dihedral angle between the two planes defined by the vectors $\mathbf{R}$ and $\mathbf{r}_{\mathrm{A}}$, and $\mathbf{R}$ and $\mathbf{r}_{\mathrm{B}}$. A standard Jacobi grid consisting of $22 R$ values in the range from 2.5 to $20.0 \mathrm{a}_{0}, 7$ Gauss-Legendre quadrature values for the angles 
$\Theta_{\mathrm{A}}$ and $\Theta_{\mathrm{B}}$ in the range from $0^{\circ}$ to $180^{\circ}$, and 6 Gauss Chebyshev quadrature values for $\Phi$ in the range of $0^{\circ}-180^{\circ}$ was applied in the PES calculations. At each $R$ value the angular dependence of the potential was represented by an expansion of the $\operatorname{CCSD}(\mathrm{T})$ interaction energy:

in the following angular functions

$$
V\left(R, \Theta_{\mathrm{A}}, \Theta_{\mathrm{B}}, \Phi\right)=\sum_{L_{\mathrm{A}} L_{\mathrm{B}} L} C_{L_{\mathrm{A}} L_{\mathrm{B}} L}(R) \cdot A_{L_{\mathrm{A}} L_{\mathrm{B}} L}\left(\Theta_{\mathrm{A}}, \Theta_{\mathrm{A}}, \Phi\right)
$$

$$
A_{L_{\mathrm{A}} L_{\mathrm{B}} L} \equiv \sum_{M=0}^{\min \left(L_{\mathrm{A}}, L_{\mathrm{B}}\right)}(-1)^{M}\left(\begin{array}{ccc}
L_{\mathrm{A}} & L_{\mathrm{A}} & L \\
M & -M & 0
\end{array}\right) \times P_{M}^{L_{\mathrm{A}}}\left(\cos \Theta_{\mathrm{A}}\right) P_{M}^{L_{\mathrm{B}}}\left(\cos \Theta_{\mathrm{B}}\right) \cos M \Phi
$$

where the associated Legendre functions are defined in terms of the usual Legendre polynomials $P_{L}(x)$ with argument $x=\cos \Theta$. The quantity in round bracket in Eq. (6.4) is the Wigner 3jsymbol.

Here, the $A_{L_{\mathrm{A}} L_{\mathrm{B}} L}$ form a set of orthonormal coupled angular basis functions and the spherical expansion coefficients $C_{L_{\mathrm{A}} L_{\mathrm{B}} L}(R)$ only depend on $R$. These expansion coefficients are obtained by projection and numerical quadrature, as for example described for $\mathrm{CO}-\mathrm{H}_{2}$ collisions in Ref. (26). This yields 140 unique expansion coefficients for the CO-CO system at each $R$ value. The expansion reproduces the potential in the region of the potential well with a standard deviation of $0.2 \mathrm{~cm}^{-1}$. At the collision energy of nearly $1500 \mathrm{~cm}^{-1}$ the standard deviation is larger, $40 \mathrm{~cm}^{-1}$, because the potential is more strongly anisotropic, but this is still a relative error of less than 3 percent.

In all trajectory calculations the Venus code version 2005(27) was used. The program was interfaced with a routine that enables the use of our representation of the interaction energies. The representation of the potential as a Legendre expansion is optimal for quantum methods using a grid of Jacobi coordinates. The Venus program requires the potential as function of Cartesian coordinates. The analytic form of the Jacobian matrix needed to also transform the potential derivatives with respect to Jacobi coordinates to derivatives with respect to Cartesian coordinates is more complicated. We therefore chose to apply numerical differentiation where for each Cartesian coordinate a left and right stepsize of $10^{-4} a_{0}$ was used. The $\mathrm{CO}-\mathrm{CO}$ intermolecular potential was supplemented with a CO monomer potential, which depends on the bond length $r$ and was represented by a Morse potential. The Morse parameters $D_{e}$ and $r_{e}$ were taken from 
experiment(28) with $D_{e}=11.24 \mathrm{eV}$ and $r_{e}=1.128 \AA$ A t the parameter in the exponent was adapted to optimally reproduce the set of rovibrational levels in the range spanned by the collision energy. Unlike the case of calculations for CO colliding with rare gas atoms where the linear dependence on intramolecular distance of the expansion coefficients was introduced, these vibrational coupling parameters were neglected in the $\mathrm{CO}+\mathrm{CO}$ collision calculations since the $\mathrm{CO}+\mathrm{Rg}$ atom collision calculations showed little effect of this intra/inter coupling. Propagation in time of the Newtonian equations of motion was performed with the Velocity Verlet algorithm with a stepsize of $2 \times 10^{-17}$ s. Initially, the collision partners were set apart with a center of mass distance of $8 \AA$. A trajectory was considered finished when the center of mass distance had again reached a value of $8 \AA$. Main calculations were performed at a mean collision energy of $1460 \mathrm{~cm}^{-1}$. Additional calculations were as well performed at higher collision energies for comparison with the trail experiments: $3600 \mathrm{~cm}^{-}$ ${ }^{1}$ for $\mathrm{CO}$ on $\mathrm{Ar}$ and $3000 \mathrm{~cm}^{-1}$ for $\mathrm{CO}$ on ${ }^{13} \mathrm{CO}$. At these higher energies relative velocities are similar to those for $\mathrm{CO}$ with $\mathrm{He}$ at $700 \mathrm{~cm}^{-1}$. For convenience in the later calculations of differential cross sections and alignment parameters, the impact parameters were controlled manually rather than by a random number generator. The maximum impact parameter values $b_{\max }$ were found by trial and error and set to $7 \AA$ corresponding to a total angular momentum value for the collision complex of 184. The impact parameter range was stepped from 0 to $b_{\max }$ with $0.1 \AA$ steps. At each impact parameter 50000 trajectories, varying in vibrational coordinate and orientation of $\mathrm{CO}$, were generated with the initial values of the rovibrational quantum numbers of $\mathrm{CO}$ set to $v=0, j=0$ and $v=0, j=1$, the latter to account for the small fractional population of $j=1$ in the molecular beams (experimental section). From the Cartesian coordinates and momenta of the atoms at the end of each trajectory the scattering angle and angular momentum vector(s) were determined. Results were binned in the scattering angle (in 40 equally spaced cosine intervals) and $j$ ' value (derived from $E_{r o t}=j^{\prime}\left(j^{\prime}+1\right) / 2 \mu r^{2}$, where $r$ is the instantaneous intramolecular distance). This binning provided both the $j$ ' dependent partial and differential cross sections. Because of the large number (millions) of trajectories needed for good statistics, the use of Gaussian binning, which should yield better results, was deemed to be too expensive. In order to find the scattering angle dependent alignment moments, the angular momentum vectors were transformed to the $\mathbf{k}-\mathbf{k}^{\prime}$ (collision) coordinate system with its z-axis along $\mathbf{k}$ and $\mathrm{x}$-axis in the $\mathbf{k}-\mathbf{k}$ ' plane. The alignment moments $A_{0}^{\{2\}}(\theta)$ and $A_{2+}^{\{2\}}(\theta)$ were calculated based on the formulas described in Table I of Ref. (29). In each cosine interval of the scattering angle, the alignment moments were determined by the averages: 


$$
\begin{gathered}
A_{0}^{2}=\left\langle 3 \cos ^{2} \theta-1\right\rangle, \\
A_{1+}^{2}=\sqrt{3}\langle\cos \theta \sin \theta \cos \varphi\rangle, \\
A_{2+}^{2}=\left\langle\sin ^{2} \theta \cos (2 \varphi)\right\rangle .
\end{gathered}
$$

Here $\theta$ is the angle that the angular momentum vector $j$ ' makes with the $\mathrm{z}$ axis, and $\varphi$ is the angle between the projection of the angular momentum vector in the collision xy plane and the (collision) $\mathrm{x}$ axis.

In quasi-classical trajectory methods there is no conservation of vibrational zero-point energy we applied a filter on the outcome of the calculations: only trajectories having a vibrational energy change less than 5\% were selected for further analysis. It turned out that lowering this criterion changed the outcome of the analysis only beyond statistical noise (with this setting $99 \%$ of the trajectories passed the selection process).

\section{Detailed Comparison of experiment and theory}

\subsection{Results and analysis at $1460 \mathrm{~cm}^{-1}$ collision energy}

Experiment and theory for ${ }^{13} \mathrm{CO}-\mathrm{CO}$ collisions at $1460 \mathrm{~cm}^{-1}$ are compared in Fig. 1 of the main text. While agreement is not perfect - there is significant experimental uncertainty, especially from the onion-peeling inversion step - the main features of the experiment including the extraordinary channel indicated by the arrow in Fig. 1, are reproduced by quasi-classical theory. While quantum theory is more accurate, QCT reveals the collision pathway, which for 'prototypical' trajectories gives the key insight into the mechanism resulting in the extraordinary forward scattering symmetric excitation (FSSE) signal indicated in Fig. 1 of the main text. Dynamics extracted from QCT were illustrated in Fig. 2 of the main text, which shows the intermolecular angles, distances and interaction energy as a function of time for a trajectory with $\left(j^{\prime}=15, j^{\prime \prime}=15\right)$ final products. The $(15,15)$ pair correlated differential cross section with forward scattering, angle 30 degrees or less, originates predominantly from trajectories with an impact parameter between 3.0 and 3.5 A. In order to elucidate the origin of the dominant forward scattering the trajectories in this impact parameter range were recalculated and the coordinates and momenta at $2.5 \mathrm{fs}$ intervals were stored. Because of storage limitations this was only possible for the first 5000 trajectories with around 600 steps at each of the 6 impact parameters. From the stored coordinates the distance between the $\mathrm{C}$ atoms $\mathrm{R}_{\mathrm{CC}}$, the distance between the 
oxygen atoms $\mathrm{R}_{\mathrm{OO}}$, the distance between the centers of mass of the two diatoms $\boldsymbol{R}$, the Jacobi angles $\theta_{\mathrm{a}}, \theta_{\mathrm{b}}$ and $\phi$, the scattering angle $\theta$, and the interaction potential $\mathrm{V}$, were calculated at each interval.

More than half of the trajectories leading to forward scattering were very similar to the prototypical trajectory sketched in Fig. 2: The molecules start in position A antiparallel with $\theta_{\mathrm{a}}$, $\theta_{\mathrm{b}}=40$ and $140 \pm 10$ degrees, respectively, and in plane, $\phi=170$ to 180 degree. In the first $300 \mathrm{fs}$ the orientations hardly change and a weakly attractive part in the PES is sampled. After $300 \mathrm{fs}$, position $B$, the $C$ atoms collide and the potential rises sharply. This induces a torque in each of the $\mathrm{CO}$ molecules such that they undergo an anti-geared motion. This is also the point where the exchange from translational to rotational energy takes place. Subsequent rotation over 110 degrees of each of the molecules in the next $200 \mathrm{fs}$ leads to an attraction when a configuration close to the dimer equilibrium geometry is reached at position C. Because of the difference in van der Waals radii, $\mathrm{r}_{\mathrm{C}}$ larger than $\mathrm{r}_{\mathrm{O}}$, the further rotation by 100 degrees in the next $100 \mathrm{fs}$ does not lead to repulsion, the two oxygen atoms just slide along each other during the rotational (and slowdown relative translational) motion to position $D$. In the remainder of the time the molecules fly further apart without change in angular momentum, positions $E, F$.

The other trajectories leading to forward scattering follow the same sequence of events: slight attraction, hit of the repulsion wall after $300 \mathrm{fs}$, induction of anti-geared rotation, passage through a potential minimum and separation. They differ from the prototypical ones mostly by having a dihedral angle around 100 degrees (this influences the alignment of the $\mathrm{j}$ vectors). The $(0,0) \rightarrow(15,15)$ energy transfer collision from $\mathrm{R}=-8 \AA$ to $+8 \AA$ at collision energy $1460 \mathrm{~cm}^{-1}$ covers a time span of $\sim 1500 \mathrm{fs}$, and the relative positions A - F of the two CO molecules are indicated in Fig. 2. The initial and final relative velocities are 796 and $492 \mathrm{~m} / \mathrm{s}$. respectively, the $\mathrm{CO}\left(j^{\prime}=15\right)$ rotational period is $\sim 560 \mathrm{fs}$ and the $\mathrm{CO}(v=0)$ vibrational period is $\sim 15 \mathrm{fs}$. Note that at $\sim 240 \mathrm{fs}$ the molecules pass a geometry that is very close to the dimer equilibrium geometry and at 500 fs the geometry is close to that of the second dimer minimum geometry. 


\subsection{QCT results at $1460 \mathrm{~cm}^{-1}$ collision energy}

$j^{\prime}=15$ is a minor $j^{\prime}$ product channel at collision energy $1460 \mathrm{~cm}^{-1}$, as can be seen in the state-tostate integral cross sections (ICS) shown in Fig. S2. While the general trend of a smooth decrease in the ICS with increasing $j$ ' seen in Fig. S2 can be represented by the energy gap law, a slight bulge at higher $j$ ' states is evident.

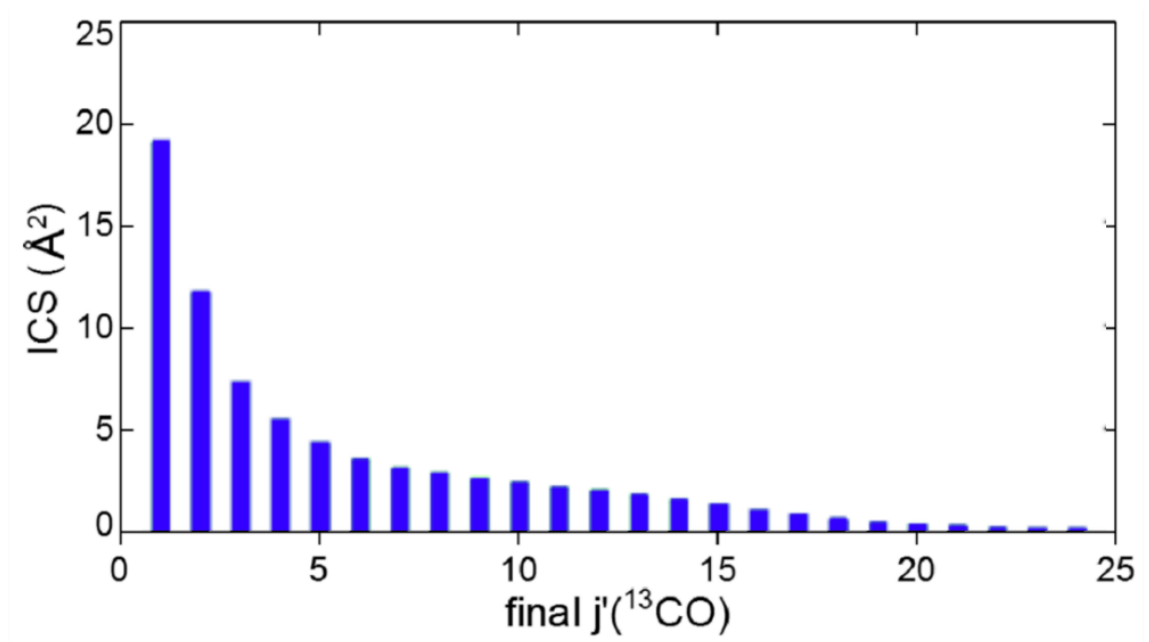

Figure S2: Integral state-to-state cross sections for ${ }^{13} \mathrm{CO}(j=0)$ to final $j$ ' quantum states, from QCT calculation at $1460 \mathrm{~cm}^{-1}$ collision energy.

Opacity functions which show the cross section for producing $(15,15)$ products as a function of impact parameter are shown in Fig. S3, and, for comparison, for $(15,5)$ products, which correspond to less rotational energy transfer than $(15,15)$. Two curves underlie the $(15,5)$ distribution; the curve peaking at lower $b$ values corresponds to the secondary rainbow and at higher $b$ values to the primary rainbow. Both contributions are also present in the $(15,15)$ data, but an additional contribution indicated by an arrow in Fig. S3a) also appears at impact parameters centered around $3.2 \AA$, which is attributed to the forward scattering symmetric excitation (FSSE) signal indicated in Fig. 1 of the main text for the $(15,15)$ channel. 


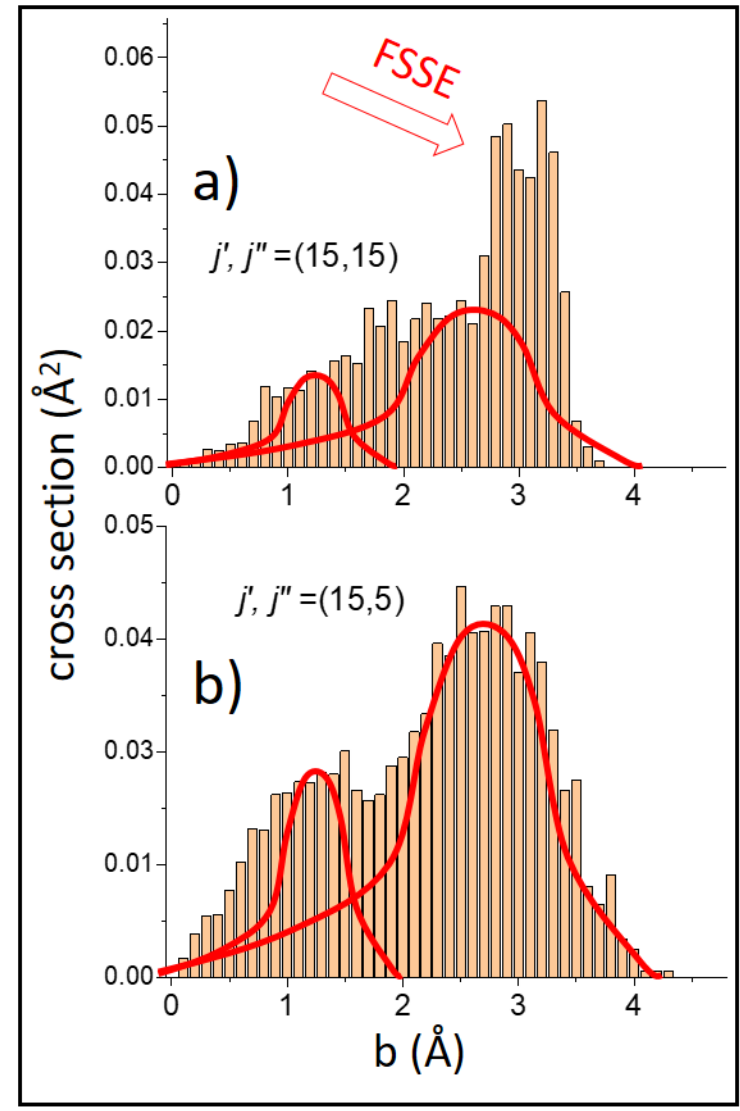

Figure S3: Contributions of different impact parameters to the relative total cross sections at $1460 \mathrm{~cm}^{-1}$ collision energy. The red curves, fit by eye to the $(15,5)$ data set and scaled to the $(15,15)$ data, indicate the range of impact parameters $b$ which result in 'normal' or atom-molecule type angular distributions of products. Impact parameter values that lead to FSSE signal are indicated by an arrow.

Deviation from EGL behavior is more evident in the pair-correlated partial ICSs shown in Fig. S4a). An extra 'bump' is evident by $j$ ' $=10$ (see also Fig. S2) and becomes quite obvious for $j$ ' = 13-16. In panel b) of Fig. S4 the $j^{\prime}=14$ ICS is shown separately along with a curve fit to the ICS by an energy gap law (EGL) model. A simple indication of the FSSE channel is given by the shaded region in Fig. S4b). The fractional integrated area of this channel compared to the EGL contribution is plotted in Fig. S4c) and indicated as symmetric excitation because it peaks for the given collision energy at the $(14,14)$ state, where roughly $20 \%$ of the $j^{\prime}=14$ signal is due to the extraordinary channel. Such a large fraction indicates, and is supported by sampling the QCT results, that collisions leading to $j^{\prime}=j{ }^{\prime}=15$ share a wide range of initial alignments with $\theta_{\square}$ from $0^{\circ}$ to $\sim 45^{\circ}, \theta_{\square}$ from $180^{\circ}$ to $\sim 135^{\circ}$, and $\phi$ ranging from $90^{\circ}$ to $180^{\circ}$. 

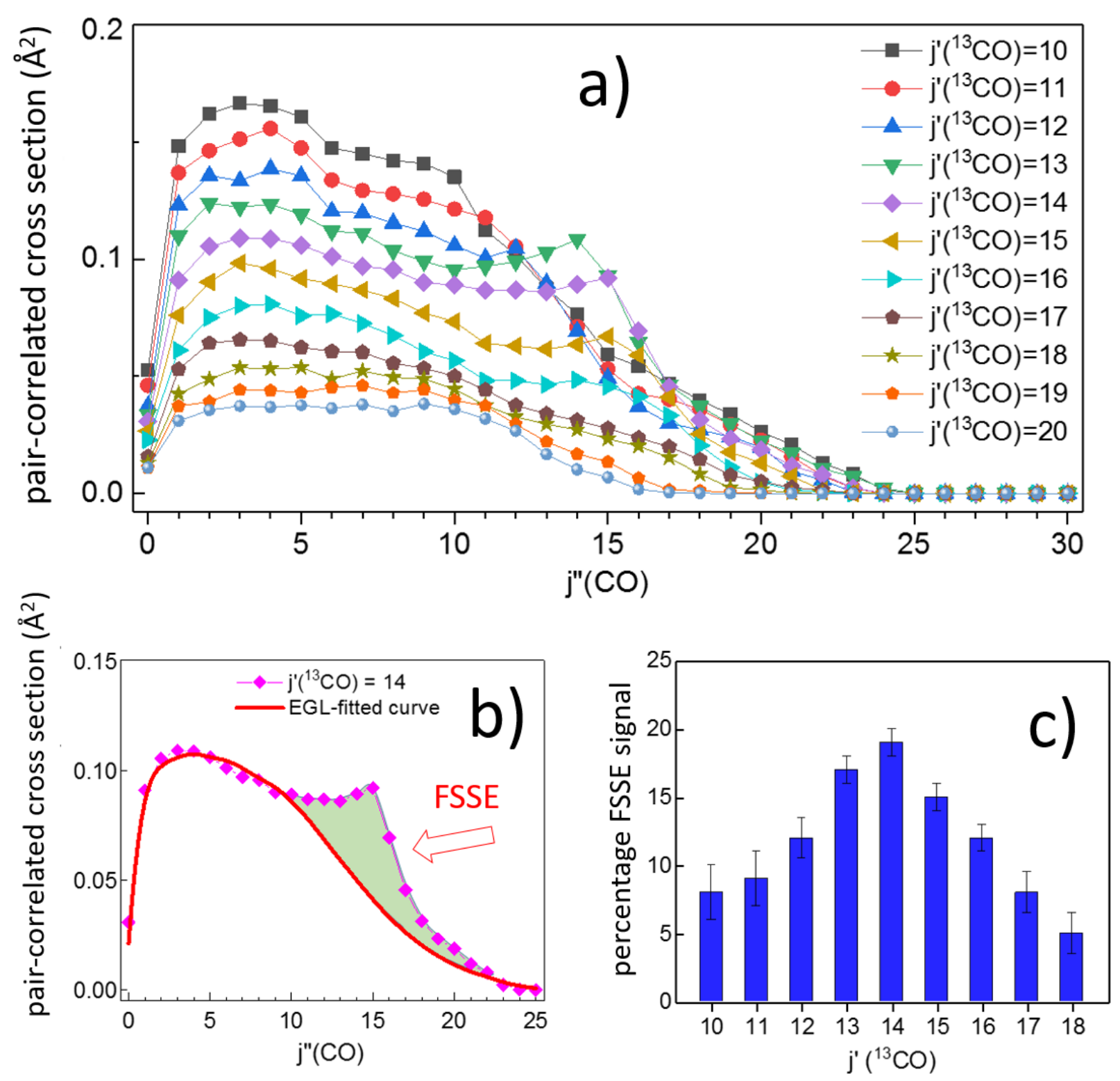

Figure S4: Panel a: Pair-correlated partial integral cross sections of rotationally inelastic CO$\mathrm{CO}$ collisions for different $j^{\prime}\left({ }^{13} \mathrm{CO}\right)$ as a function of the $j{ }^{\prime \prime}(\mathrm{CO})$ final rotational state at $1460 \mathrm{~cm}^{-1}$ collision energy. Panel b: The partial pair-correlated cross sections for $j{ }^{\prime \prime}\left({ }^{13} \mathrm{CO}\right)=14$ with deviation from the energy gap law fitted curve in the region of forward scattered symmetric excitation (FSSE). Panel c: percentage of the FSSE signal compared to energy gap law signal, where the error bars reflect the uncertainly of the EGL fit to the data shown in panel b). 
Pair correlated partial differential cross sections for CO-CO collisions, Fig. S5, confirm the remarkable forward scattering for the extraordinary product channel with symmetric high $\left(j{ }^{\prime} \sim j^{\prime \prime}\right)$ excitation. The most strongly forward scattering occurs for exactly symmetric excitation $\left(j{ }^{\prime}=j{ }^{\prime \prime}\right)$. The effect is even stronger (i.e., exclusively forward scattering) when selecting only initial impact parameters $b \sim 3 \AA$, the position of the 'extra' peak in the opacity function shown in Fig. S3. For comparison, panel b) of Fig. S5, a similar QCT calculation for scattering of ${ }^{13} \mathrm{CO}$ with $\mathrm{N}_{2}$ does not show forward scattering or enhanced signal for the $(15,14)$ and $(15,16)$ pairs, in accord with the raw data shown in Fig. S1 and the contour plot shown in Fig. 1 in the main text Panel c) shows results for scattering with a fictitious ${ }^{14} \mathrm{C}^{14} \mathrm{O}$, which shows similar scattering properties as ${ }^{12} \mathrm{C}^{16} \mathrm{O}$. This indicates that kinematic effects due to the displacement of center of mass of CO compared to $\mathrm{N}_{2}$ are minor. 


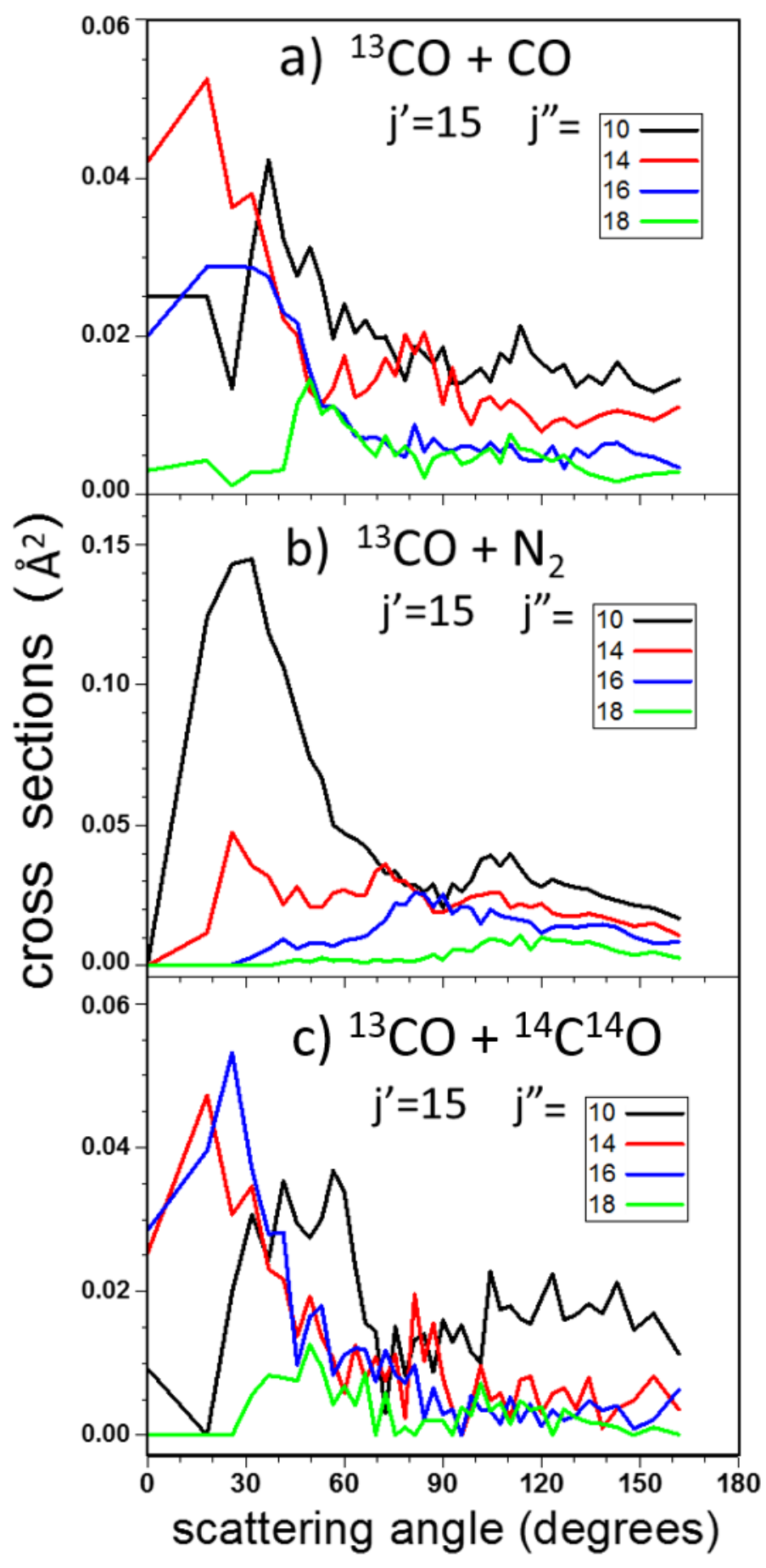

Figure S5: QCT results for pair correlated partial DCSs at $1460 \mathrm{~cm}^{-1}$ collision energy for scattering of a) ${ }^{13} \mathrm{CO}(j=0)+\mathrm{CO}(j=0)$, and b) ${ }^{13} \mathrm{CO}(j=0)+\mathrm{N}_{2}(j=0)$ and c) ${ }^{13} \mathrm{CO}(j=0)+$ ${ }^{14} \mathrm{C}^{14} \mathrm{O}(j=0)$ to produce ${ }^{13} \mathrm{CO}\left(j^{\prime}=15\right)$ and the indicated $j$ " partner. The fictitious molecule ${ }^{14} \mathrm{C}^{14} \mathrm{O}$ is introduced in $\mathrm{c}$ ) in order to illustrate that a non-shifted center-of-mass yields similar results to those shown in panel a).

With the present apparatus it was not possible to explore collision energies much lower than $1460 \mathrm{~cm}^{-1}$ reported here. Instead, QCT calculations at were carried out at $500 \mathrm{~cm}^{-1}$ in order to 
determine if the key characteristics of the forward scattering symmetric excitation (FSSE) channel found at $1460 \mathrm{~cm}^{-1}$ are present at lower collision energy. This is indeed confirmed by the QCT results presented in Fig. S6. Another motivation was to connect our present results with predictions by Ref. (18) where fully quantum theory was applied to CO-CO collisions at lower collision energy. Because of strong disagreements in collision cross sections that were found between QCT and the work of Ref. (18), fully quantum calculations were also carried out at $500 \mathrm{~cm}^{-1}$, as described in the following section.
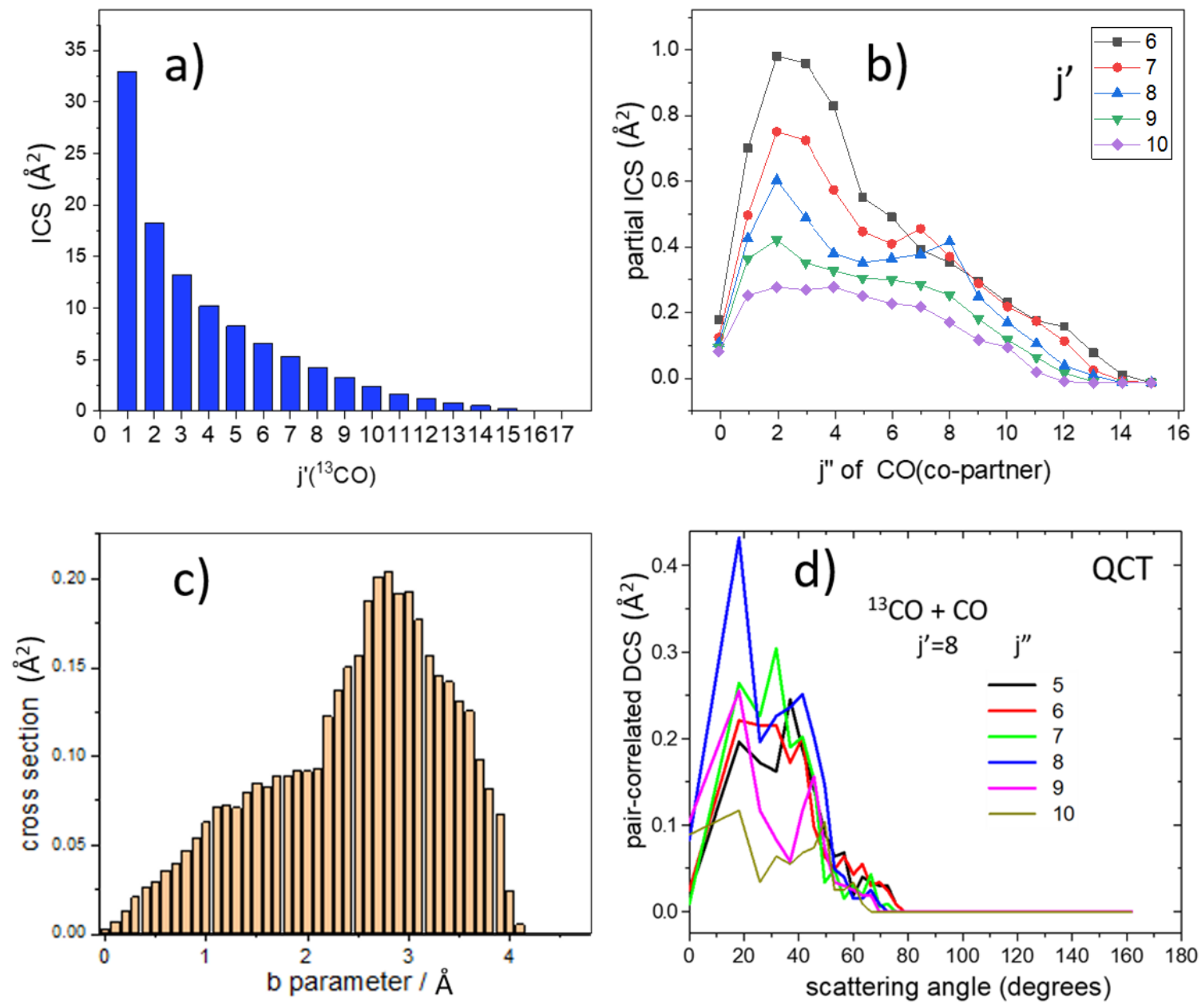

Figure S6: Results from QCT for ${ }^{13} \mathrm{CO}-\mathrm{CO}$ collisions at $500 \mathrm{~cm}^{-1}$ collision energy.

\subsection{QCT results at $500 \mathrm{~cm}^{-1}$ collision energy}

QCT results at $500 \mathrm{~cm}^{-1}$ collision energy, shown in Fig. S6, confirm the trends observed for ${ }^{13} \mathrm{CO}-\mathrm{CO}$ collisions at $1460 \mathrm{~cm}^{-1}$. Panel a) presents the state-to-state ICSs, which in accord with the EGL tail off much faster than at the higher collision energy and show a less perceptible 
increase around $j^{\prime}=8$. The increase is more apparent in the pair-correlated partial ICSs shown in panel b), where an obvious 'bump' is clear for the $(8,8)$ channel. Opacity functions (panel c) for the $(8,8)$ channel compared to neighboring channels show an extra contribution peaking at $\sim 3 \AA$, and the pair correlated partial DCSs with $b=3 \AA$ (panel d) show maximum forward scattering for the $(8,8)$ channel. Note that both the $(8,8)$ channel at $500 \mathrm{~cm}^{-1}$, and the $(14,15)$ channel 1460 $\mathrm{cm}^{-1}$ correspond to $55 \%$ transfer of translation to rotation energy, and in both cases the FSSE channel is accessed by relatively small $(\sim 3 \AA)$ impact parameters.

\subsection{Fully quantum results at $500 \mathrm{~cm}^{-1}$ collision energy}

Calculations were performed using the quantum-mechanical close-coupling (CC) approach for the initial state $(0,0)$ at collision energy of $500 \mathrm{~cm}^{-1}$. Following an expansion of the nuclear wavefunction in terms of angular functions of the $\mathrm{CO}$ monomers, the coupled equations were solved with a renormalized Numerov propagator using a home-made code on a grid of 173 intermolecular distances in the range 4-40 $a_{0}$. The coupled equations can be solved independently for each value of the total angular momentum obtained when coupling the angular momenta of the $\mathrm{CO}$ molecules, $\boldsymbol{j}_{\boldsymbol{a}}$ and $\boldsymbol{j}_{\boldsymbol{b}}$, with the angular momentum for the relative motion $\boldsymbol{L}: \boldsymbol{J}=\boldsymbol{j}_{\boldsymbol{a}}+\boldsymbol{j}_{\boldsymbol{b}}+\boldsymbol{L}$. At a collision energy of $500 \mathrm{~cm}^{-1}$, total angular momentum values up to $J_{\max }=250$ were necessary in order to converge the cross sections. The angular basis set included all rotational states up to $j_{\max }$ $=11$ for both monomers. While this does not allow to fully converge the cross section for all transitions, larger angular basis sets led to prohibitive calculations in terms of computational time as well as memory requirements. The convergence of the cross sections as a function of the size of the angular basis set was studied, and we found that the cross sections for the final states with final rotational states below $j$ ' or $j "=9$ were accurately described. Moreover, additional calculations were performed at a collision energy of $250 \mathrm{~cm}^{-1}$. In that case, the cross sections were found to be converged and showed a qualitatively similar behavior as for energy of $500 \mathrm{~cm}^{-1}$. It should be noted that our CC calculations were carried out by assuming a collision between two identical molecules, as this allows reduction of the number of coupled equations and therefore use 


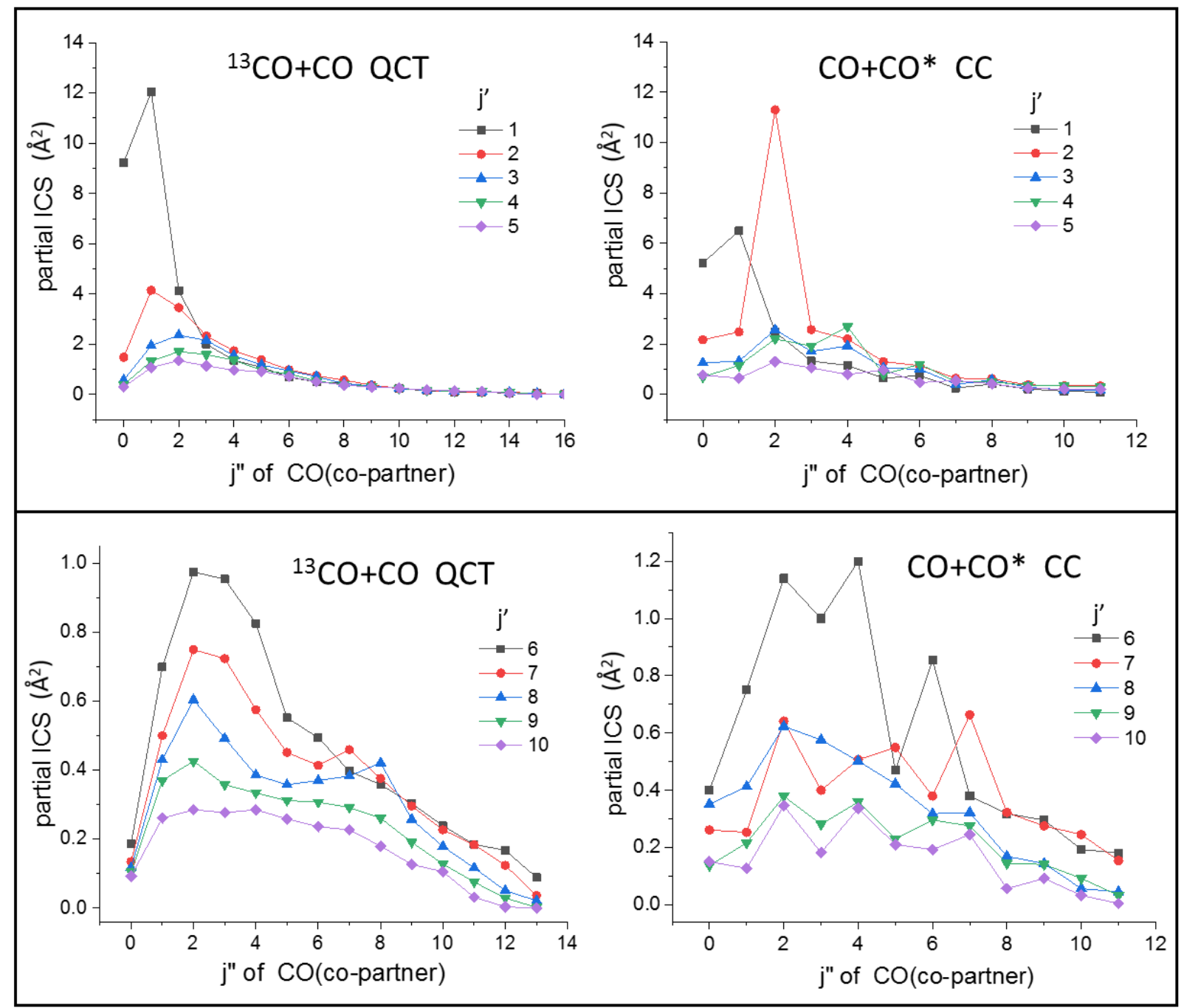

Figure S7: Comparison of partial integral cross sections for product pairs (j',j") using QCT versus fully quantum $\mathbf{C C}$ at $500 \mathrm{~cm}^{-1}$ collision energy, with correction for non-identical molecules.

of a larger angular basis set. This approach amounts to neglecting the small difference between ${ }^{12} \mathrm{CO}$ and ${ }^{13} \mathrm{CO}$, which is not expected to affect the present results dramatically.

The CC cross sections were found to agree well with the QCT results. An important difference arises from the fact that the CC calculations predict a propensity for transitions to final states ( $j$, $j$ ') with $j$ ' or $j$ " even, as shown in Fig. S7, where QCT for ${ }^{13} \mathrm{CO}-{ }^{12} \mathrm{CO}$ is compared with CC results for ${ }^{12} \mathrm{CO}+{ }^{12} \mathrm{CO}$, where this can be explained by the fact that the $\mathrm{CO}$ molecule behaves similarly to a homonuclear diatomic molecule, for which only transitions $\Delta j=$ even are allowed. The dominant transition from the initial state $(0,0)$ is found to be to the $(2,2)$ state. It should be noted that the agreement between the CC and QCT is expected to increase with increasing collision energy. The 
present CC calculations thus validate the QCT calculations. On the other hand, large discrepancies are observed compared to the quantum-mechanical results of Ref. (18), which reports similar calculations. Those calculations do not appear to be fully converged due to the much smaller basis sets $\left(j_{\max }=7\right)$ and a smaller number of total angular momentum values.

Ref. (18) reports state-to-state ICSs for CO-CO collisions over the $0-1000 \mathrm{~cm}^{-1}$ collision energy range, using the MOLSCAT program with an accurate PES (30). Our values for partial ICSs from $(0,0)$ to the $j^{\prime}=3$ state (the highest states reported in (18)) are compared in Table S1.

Table S1: Partial integral cross sections at $500 \mathrm{~cm}^{-1}$ collision energy for QCT and CC (this work) compared to previous quantum calculation. For final states with $j^{\prime}=3$, Ref (18) predicted the transition to the state $(3,3)$ to be dominant due to an error of a factor of two. In addition, the calculations of Ref (18) may not be fully converged.

\begin{tabular}{|l|l|l|l|l|}
\hline $500 \mathrm{~cm}^{-1}$ & $j^{\prime}, j^{\prime \prime}$ & Ref. $(18)$ & QCT & CC \\
\hline $0,0 \rightarrow$ & 3,4 & $4 \AA^{2}$ & 1.6 & 1.9 \\
\hline & 3,3 & 7 & 2.2 & 1.7 \\
\hline & 3,2 & 5 & 2.5 & 2.6 \\
\hline & 3,1 & 2.5 & 2.0 & 1.3 \\
\hline
\end{tabular}

CC results for pair-correlated DCSs at $500 \mathrm{~cm}^{-1}$ collision energy shown in Fig. S8 for the $j^{\prime}=8$, $j ”=5-9$ final states confirm strongly forward scattering predicted by QCT in Fig. S6d. 


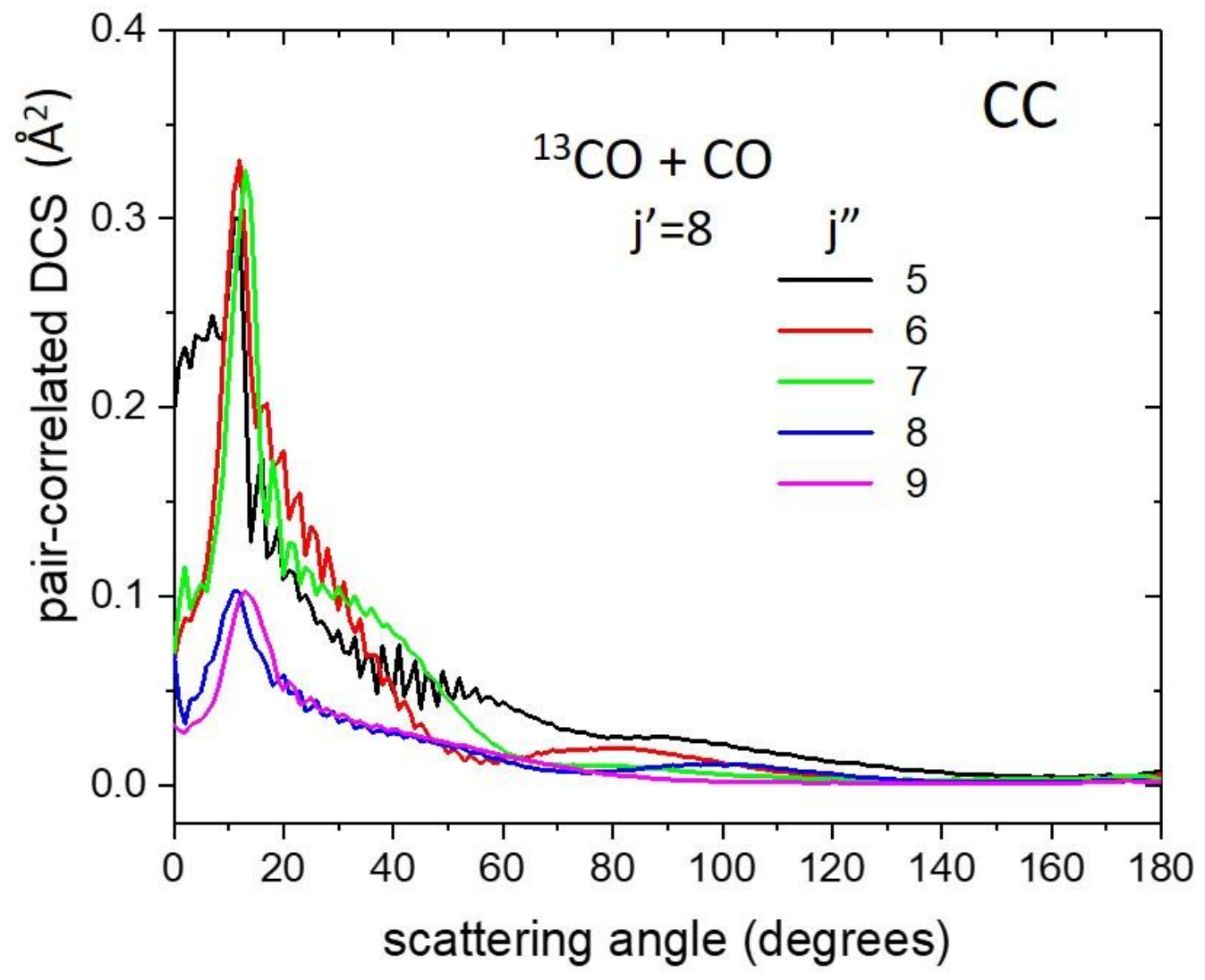

Figure S8: Pair-correlated differential scattering cross section from fully quantum CC calculation for excitation to the $j^{\prime}=8, j^{\prime \prime}$ states at $500 \mathrm{~cm}^{-1}$ collision energy. 
Data and materials availability: All data are available in the main text or the supplementary materials, as well as online at Dryad (31).

\section{Caption for Data S1:}

Data S1: excel format file for all Figures in the main text and supplementary materials.

\section{Caption for Movie files M1 and M2}

VMD (19) visualizations of two types of trajectories for CO-CO collisions at $1460 \mathrm{~cm}^{-1}$ collision energy are shown, all begin at $\mathrm{R}=8.0 \AA$ : The larger $\mathrm{C}$ atom (van der Waals radius $1.7 \AA$ ) and smaller $\mathrm{O}$ atom $(1.5 \AA)$ are shown in blue and red colors, respectively. The movie step size is $2.5 \mathrm{fs} /$ frame.

M1 shows seven typical FSSE trajectories with $\left(j^{\prime}, j{ }^{\prime \prime}=15,15\right)$ with the listed initial conditions in the following sequence:

$\begin{array}{lllllll}\mathrm{b}(\AA) & \mathrm{R}_{\mathrm{CC}}(\AA) & \mathrm{R}_{\mathrm{OO}} & \theta_{\mathrm{a}}(\text { degrees }) & \theta_{\mathrm{b}} & \phi & \theta \\ 3.1 & 7.205 & 8.696 & 45.256 & 130.630 & 178.583 & 21.4 \\ 3.1 & 7.155 & 8.728 & 40.524 & 131.509 & 168.657 & 15.1 \\ 3.2 & 7.191 & 8.704 & 44.514 & 131.366 & 178.599 & 16.9 \\ 3.2 & 7.302 & 8.635 & 50.220 & 123.767 & 170.095 & 18.2 \\ 3.2 & 7.368 & 8.584 & 45.403 & 113.907 & 175.970 & 22.8 \\ 3.3 & 7.288 & 8.645 & 49.494 & 124.533 & 169.958 & 15.4 \\ 3.3 & 7.130 & 8.745 & 38.980 & 132.973 & 168.455 & 13.6\end{array}$

and M2 shows eight typical trajectories resulting in $\left(j^{\prime}, j "=15,5\right)$ with the listed initial conditions in the following sequence:

$\begin{array}{lllllll}\mathrm{b}(\AA) & \mathrm{R}_{\mathrm{CC}}(\AA) & \mathrm{R}_{\mathrm{OO}} & \theta_{\mathrm{a}} \text { (degrees) } & \theta_{\mathrm{b}} & \phi & \theta \\ 2.8 & 7.997 & 8.077 & 128.030 & 133.628 & 99.612 & 60.4 \\ 2.8 & 8.122 & 7.906 & 95.558 & 84.600 & 5.183 & 54.0 \\ 2.8 & 7.919 & 8.182 & 114.566 & 131.887 & 156.991 & 54.7 \\ 2.8 & 7.597 & 8.359 & 30.774 & 78.399 & 127.005 & 67.9 \\ 2.8 & 7.583 & 8.373 & 24.910 & 79.008 & 158.161 & 65.9 \\ 2.8 & 8.464 & 7.665 & 130.299 & 85.864 & 41.119 & 57.3 \\ 3.0 & 7.460 & 8.473 & 24.033 & 88.529 & 153.003 & 64.2 \\ 3.0 & 7.560 & 8.390 & 23.381 & 80.371 & 158.644 & 54.8\end{array}$

